\title{
INTERNET OF THINGS (IOT) FOR SMART PRECISION FARMING AND AGRICULTURAL SYSTEMS PRODUCTIVITY: A REVIEW
}

\author{
Lalit Kumar ${ }^{1}$, Prasant Ahlawat ${ }^{2}$, Pradeep Rajput ${ }^{3}$, R.I Navsare ${ }^{4}$, Pradeep Kumar Singh $^{3}$ \\ ${ }^{1}$ Indian Institute of Farming System Research (IIFSR), Modipuram, Meerut, U.P., India \\ ${ }^{2}$ Department of Plant Pathology, C.C.S University Campus, Meerut, Uttar Pradesh, India \\ ${ }^{3}$ Department of Agronomy, 4Department of Soil Science, Sardar Vallabhbhai Patel University of Agriculture \& \\ Technology, Meerut, Uttar Pradesh, India
}

\begin{abstract}
The Internet of Things (IoT) is a promising technology which offers effective and reliable solutions for multi-domain modernization. In order to automatically manage and track agricultural farms with minimal human intervention, IoT-based solutions are being created. There are several aspects of innovations involved in the field of IoT in agriculture in the review paper. It describes the key components of precision farming based on IoT. A detailed discussion on network technologies used in IoT-based agriculture, including network architecture and layers, network topologies and protocols, was presented. In addition, the relation of agricultural systems based on IoT with related technologies like cloud computing, storage of big data and analytics was also presented. Furthermore, protection concerns have been illustrated in IoT agriculture. A list of applications based on smart phones and sensors built for various aspects of farm management has also been presented. In addition, the introduction of precise agriculture, which is an alternative to future agriculture, allows for the prediction of supply and demand by means of convergence technology; real-time monitoring and quality maintenance over the life cycle of agricultural products. To increase the productivity of their day-to-day jobs, farmers have already begun to employ some high-tech farming techniques and technologies. Sensors installed in fields, for instance, allow farmers to obtain detailed maps of both the area's topography and resources, as well as variables such as soil acidity and temperature.
\end{abstract}

To predict weather conditions in the coming days and weeks, they can also access climate forecasts. Farmers can remotely track their equipment, crops, and livestock using their smart phones, as well as collect stats on their feeding and producing livestock. They can also use this technology for their crops and livestock to run statistical predictions. And drones have become an invaluable instrument for farmers to survey their lands, conduct field research, and produce data in real time. Both of these techniques lead to precision farming or precision farming, the method of using satellite imagery and other technologies (such as sensors) to observe and record data in order to increase production performance while reducing costs and maintaining resources.

Keywords - Precision farming; IoT technologies; artificial intelligence; machine learning

\section{INTRODUCTION}

Major innovations have been made in human history to increase agricultural yields with less capital and labour efforts. Nevertheless, during all these periods, the high population rate never allows demand and supply to balance. According to estimated estimates, the world population is predicted to hit 9.8 billion by 2050 , a rise of around 25 percent from the current figure. Among developed countries, almost the entire aforementioned population growth is expected to occur. On the other hand, the urbanisation trend is projected to continue at an accelerated pace, with approximately 70 percent of the world's population expected to be urban by 2050 (currently 49 percent). In addition, income levels will be multiples of what they are now, driving food demand higher, especially in developing countries. As a result, their diet and food quality would be more cautious for these nations; thus, consumer tastes will shift from wheat and grains to legumes and, later, to meat. Food production should double by 2050 in order to feed this bigger, more populated, and wealthier population. In particular, the current figure of 2.1 billion tonnes of annual cereal production is expected to hit approximately 3 billion tonnes, and annual meat production is expected to rise by more than 200 million tonnes to meet 470 million tonnes of demand (Abhishek, et al., 2019). In fact, crops such as cotton, rubber, and gum play important roles in the economies of many nations, not only for food, but also for crop production. In addition, the bio-energy demand dependent on food crops has recently started to increase. Even before a decade ago, only 110 million tonnes of coarse grain (approximately 10 percent of world production) were used for ethanol production (Elder and Hayashi, 2018). Food security is at risk because of 
the growing use of food crops for bio-fuel production, bioenergy, and other industrial uses.

The consequence of these demands is a further increase in pressure on already limited agricultural resources.

As a whole, there are numerous implementations, protocols and prototypes in the agricultural sector. Research trends in IoT agriculture include network platforms, network design, applications, security, and challenges, including (Jayaraman et al., 2016). In addition, numerous IoT policies and guidelines in the agricultural sector have been introduced in several countries and organisations worldwide. However, a fair amount of work has been done in the IoT agricultural environment and there is a need for comprehensive IoT analysis in the sense of agriculture to understand the current status of science. This paper analyses various issues and trends in IoT smart farming in order to transform agricultural technologies through IoT innovation.

\section{PRECISION FARMING}

Precision farming uses a number of technologies to maximise crop yields, including GPS systems, sensors and Big Data. ICT-based decision support systems, backed up with real-time data, may also provide information on all aspects of farming at a degree of granularity not previously possible, rather than replacing farmers' experience and gut instinct. This makes it easier to make better choices, resulting in less waste and maximum productivity in operations. Computer-based imaging, GPS technology, science-based solutions, climate forecasting, technical solutions, environmental controls and more are among the disciplines and skills now needed for agriculture.

\section{INTERNET OF THINGS}

IoT can be interpreted as a network of intelligent, interconnected devices that are able to communicate with each other and produce relevant data about the world in which they operate. In the sense of IoT, practically any computer capable of establishing an Internet link may therefore be considered a "thing," such as household appliances, electronics, furniture, agricultural or industrial machinery and even individuals (Madakam et al., 2015).

While the concept of IoT is not new, its adoption has increased in recent years, primarily due to the advancement of technologies that support it, including hardware enhancement, with the resulting decrease in size and power consumption, improvements in Internet connectivity and between devices through wireless connection, cloud computing, artificial intelligence and big data. All of these technical components help to create a network of devices capable of data and knowledge exchange and effectively operating on the basis of network inputs (Zhao et al., 2010). Verdouw et al. (2019) stated that the architecture of IoT systems is identical to the architecture of other computer systems, but the particularities of this model must be taken into account, such as device restricted computing capacities, remote object recognition, detection and control.

\section{Agriculture Supports the IoT Towards Smart Farming}

The IT scheme at the other end of the supply chain, the decision support system at the back office, is what makes precision agriculture unique. While the technology is still in its infancy, the idea of 'the linked farm' is getting closer, farming activities in particular are somehow linked not only to each other, but also to a variety of historical data such as weather events, climate, economics, product details and specifications, system settings, etc. This is what the IoT is all about, linking processes so that agricultural practises can be interpreted in an interconnected, multidimensional way, allowing a deeper understanding of how the entire ecosystem functions. Precision agriculture will become 'decision-making' or 'smart agriculture.'

Study and research on the internet of things in terms of technical levels and structures is carried out on the basis of the latest creation of the internet of things and on the basis of the available technology analysis of the internet of things. Starting from three aspects, data collection, network service, date fusion and computation, respectively, the study of technologies such as RFID, ZigBee, sensors, cloud computing and so on is carried out, based on which the internet of things technical system architecture is further developed. In addition, study and research on the system's sensor nodes, analysis and discussion was carried out on the different technologies involved. Internet of Things refers to a network that enables a range of intelligent activities such as identification, positioning, tracking, monitoring and management by connecting devices such as RFID, Smart Sense, GPS (Global Positioning System) etc. to wireless network objects through interfaces to provide intelligence to objects, thus realising contact and dialogue between humans and objects as well as objects and objects.

\section{Future of Farming: IoT, Agricultural Sensors, \& Drones for Farming}

Smart agriculture and precision farming are beginning to take off, but they may only be the precursors to the world of agriculture's much greater use of technology. The growth of block chain technology is making its way to the IoT, and because of its ability to provide companies with valuable crop data, it could be important in the agricultural sector. Farmers may use sensors to collect crop data written on the block chain, which includes identification factors, salt and sugar content, and $\mathrm{pH}$ levels.

There will be almost 12 million agricultural sensors installed globally by 2023 for Insider Intelligence ventures. In addition, the tech giant IBM reports that half a million data points per day can be produced by the average farm, helping farmers to boost yields and increase income. Drones can spray fertilizer 40 to 60 times more quickly than they do by hand. It is 
understandable that farmers are increasingly turning to agricultural drones and satellites for the future of farming, considering all the possible advantages of these IoT applications in agriculture. Drones allow farmers to track how far crops are in their respective periods of growth. In addition, to bring them back to life, farmers may spray ailing crops with substances through drones. Drone Fly reports that 40 to 60 times faster than doing so by hand, drones will spray fertilizer.

\section{Looking to learn more?}

The future of agricultural technology is to collect and analyse large-scale agricultural data in order to optimise operating productivity and minimise labour costs. With the IoT, though, there are many more developments to consider, and the Internet of Things will impact far more sectors than just agriculture. They do not automatically think about agriculture as most people think about the internet. Most people worry about their computers or their mobile phones instead. Do people not realise that the Internet of Things (IoT) technology enables farmers to link devices to the internet in order to boost farming operations? IoT technology enables farmers to use the internet to minimise waste, improve control of pests, streamline the management of livestock and increase productivity.

\section{WASTE REDUCTION}

Farmers are continually searching for ways to decrease waste, with limited resources available for growing crops or raising livestock. Conservation of resources, particularly water, is of great importance, especially in California, a drought-stricken state. While conventional conservation methods such as irrigating only after dusk can reduce waste, the technology of the Internet of Things (IoT) can reduce waste and conserve resources even more. Smart linked sensors that are embedded in the soil, for instance, can measure moisture and PH levels. In order to ensure optimum conditions for the crops to grow, these sensors linked to smart irrigation and smart fertiliser systems can then apply just the right amount of fertiliser and water.

\section{Better Management of Pests}

With more fresh food and less pesticide use being pushed by the public, farmers are gradually looking to decrease or even reduce the use of pesticides. Sensors and cameras connected to the internet would enable farmers to track pest populations better. In the event of pest populations reaching points where crop yields are harmful, farmers can remotely release pheromones without using synthetic pesticides to control the pest population.

\section{Boost efficiency}

Farmers must increase productivity in order to maximise yields and profits. The technology of the Internet of Things
(IoT) helps farmers to become more efficient in a number of ways, such as tracking farm machinery. From tractor fleets to grain conveyor belts, IoT technology will allow farmers to track their equipment. Internet-connected sensors, for example, can be built into tractors to determine if the tractor operates at peak performance. If the tractor does not work at the highest level of performance, the sensor will send a warning to the farmer to allow urgent repairs. This will help avoid unexpected malfunctions in the tractor which will make it more efficient and longer in the field. In addition, internet sensors can be built into grain conveyor belts. These sensors are capable of measuring a number of parameters, such as belt friction. The sensing device will produce a signal that the operator can use more lubricants when the grain belt produces too much friction. A proactive maintenance approach will again allow the equipment to be used for long periods, resulting in higher productivity.

\section{AGRICULTURAL ADVANTAGES OF THE USE OF IOT}

The implementation of the Internet of Things in agriculture, as in other sectors, promises previously inaccessible productivity, resource and cost reduction, automation and data-driven processes. In agriculture, however, these advantages do not serve as upgrades, but rather as solutions to a number of dangerous problems facing the entire industry.

1. Excellence in efficacy: Farming today is in a sprint. Farmers need to develop more soil products, decrease availability of the land and increase climate variability. Farmers can track their commodity and conditions in real time via IoT-enabled agriculture. You will easily gain perspectives, forecast problems before they arise and make educated choices about how to stop them. In addition, the automation of IoT solutions, for example demand-based irrigation, fertilisation and robot selection, is applied in agriculture.

2. Enlargement: By the time we've got 9 billion people on the planet, $70 \%$ of them are going to live in urban areas. Greenhouses and hydroponic systems based on IoT allow for a short food supply chain and should be able to feed fresh fruits and vegetables to these individuals. In supermarkets, on skyscrapers' walls and rooftops, in shipping containers and, of course, in the comfort of everyone's home, smart closed-cycle agricultural systems allow food to grow practically everywhere.

3. Decreased resources. Many Ag IoT solutions are based on maximising the use of water, electricity, land resources. IoTbased precision farming relies on data collected from various field sensors that enables farmers to precisely distribute just enough resources within one plant.

4. Process cleaner: The same applies to pesticides and fertilisers as well. Not only do IoT-based precision farming systems help farmers save water and resources and thereby make farming greener, but they also reduce the use of pesticides and fertilisers considerably. Compared to 
conventional farming practises, this technique enables a healthier and more organic end product to be obtained.

5. Versatility: The increased agility of the systems is one of the advantages of using IoT in agriculture. Thanks to real-time monitoring and prediction systems, farmers are able to react quickly to any major changes in temperature, humidity, air quality and the health of each crop or soil in the field. New capabilities help agricultural professionals save crops in the light of extreme weather changes.

6. Enhanced quality of goods. Data-driven farming allows both more and better goods to expand. Farmers better understand comprehensive dependencies between the conditions and the quality of the crops by using soil and crop sensors, aerial drone monitoring and farm mapping. They can replicate the best conditions using connected systems and improve the nutritional value of the goods.

\section{PlATFORM FOR IOT AGRICULTURAL NETWORK}

The platform of the IoT agricultural network applies to both the model of big data analytics and the model of the cloud.

\section{1) The Analytics of Big Data}

Big data analysis was implemented to find out from the vast amount of data from various data formats the necessary and relevant information. Models for the control of crop diseases and crop growth are based on farm data. Big data analysis also provides agricultural producers with decision support services for crop production and optimum cost analysis (Farooq et al., 2019). The IoT agricultural network platform focused on big data analytics is shown in Figure 1.

Six components of the proposed network platform are: I Farmer / User interface, ii) Big Data processing, iii) Sensing and tracking, iv) Storage facilities, v) Protocols for communication, and vi) Physical implementations. This platform offers access to the IoT backbone and helps to collect data on soil fertility, weather conditioning, moisturization, tracking of crops online, etc.

\section{a: Experience with Farmers}

Farmer experience layer is designed to help farmers track crop productivity in multiple ways, such as by recognising the appropriateness of fertile selection, for successful growth of crops farmers are awarded. Climate conditions, conditions for crop growth, soil quality or monitoring of cattle health help farmers track the state of their company and mitigate lower output risks.

\section{b: Predictive analysis}

Through the combination of smart agricultural market intelligence and IoT technology, this study makes the whole ecosystem smarter. The major task of predictive analysis is to evaluate, explore and process digital knowledge of agricultural information. In order to verify the probabilistic probability of crop productivity until the next season, predictive analysis is performed. In order to check crop disease, weather conditions, and estimate profit / loss on the basis of crop productivity, various detecting devices are used here. Predictive analysis makes it easier for the farm to consider the best planting \& harvesting period and different strategies of farm management.

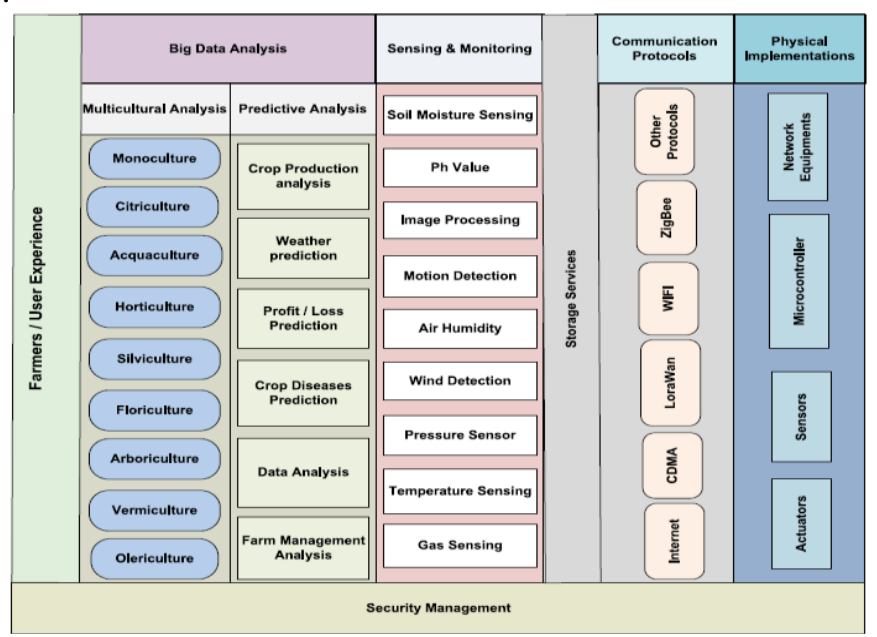

Fig. 1: Large Data Analysis-based IoT agricultural network platform

\section{c: Multicultural Evaluation}

Big data processing in this analysis eliminates, according to scientific methods, the probability of crop damage. Big data is given to improve the growth rate of water featured by the Botanic Aquaculture sheet. Other multicultural techniques such as citriculture, horticulture and floriculture, when allowed by big data analysis, have direct benefits. It is useful for seasonal growth and pest control related to crop or plant decision-making. For earthworm cultivation, vermiculture is used. Basically, arboriculture is used for the growing of woody plants. Olericulture is an application used to predict and calculate the growth rate of various vegetables plants.

\section{d: Tracking AND SENSING}

Study of sensing and monitoring is carried out by applying various instruments for sensing and monitoring. Sensors sense data and store knowledge about crop diseases. Data processed by multiple resources is automatically collected via the sensing layer. In order to monitor the disease, statistical research has been carried out on data collected from sensors. Through the web and message service, farmers obtain required information such as $\mathrm{pH}$ value, temperature, soil moisture and humidity. Data tracking of real-time images and video allows the farmer to obtain timely and reliable information.

\section{e: Protocols of Contact}

Agricultural data is gathered and encapsulated by contact protocols. The nerve centre of IoT in agriculture has been considered to be the collection and transmission of data 
through the use of these protocols. These protocols consist of technologies such as WIFI, LoraWan and Code Division Multiple Access (CDMA) technologies connected to the Internet. When third-party service providers such as LongTerm Evolution (LTE), CDMA or Global System for Mobile (GSM) are not available, ZigBee is considered to be the key enabler for communication over long distances.

\section{f: Facilities for Storage}

In order to better analyse in the future and use stored information for more efficiency in multiple seasons, farmers store crop-related information.

\section{g: Physical Commentaries}

To track various agricultural applications, several sensors, various types of actuators and microcontrollers are physically implemented. Many other network appliances, such as switches, routers and gateways, are also included in the physical layer. Entire environmental conditions are sensed at this layer and then function according to a predefined instruction.

\section{2) Cloud Infrastructure}

The cloud offers a vast amount of storage through large virtualized servers that are linked together to take the necessary action. A cloud-based IoT concept was proposed for precision farming by (Khattab et al., 2016). IoT methods are used to analyse and manage farm data through sensors and devices to produce decision-making information.

Cloud-based architecture guidelines for the IoT agricultural network are shown in Figure 2. Centered on four layers, which are Cloud Storage, Gateway, Fog Computing and hardware modules, the platform has been proposed. The cloud storage layer centerlizes all agricultural data such as temperature, soil, fertilisation, crop and agricultural marketing in the cloud and provides networked infrastructure with on-demand services. Web applications and analytics tools are often built on the cloud or web available via cloud services.

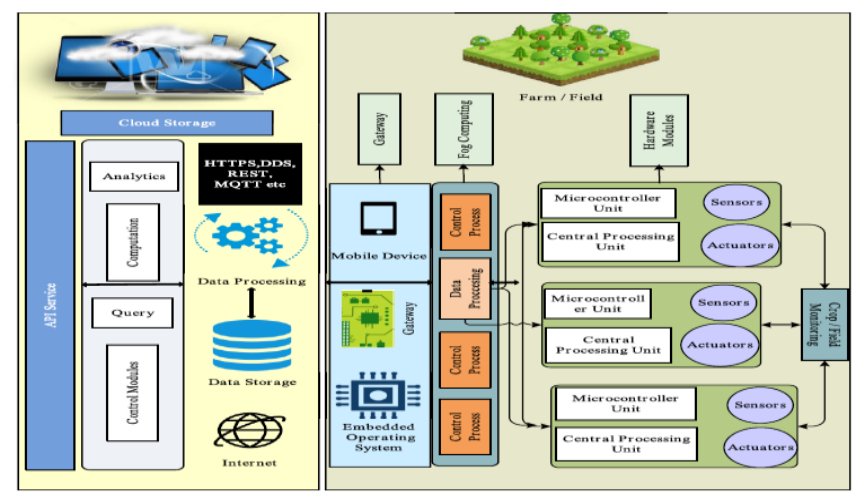

Fig. 2: Cloud-based IoT Agricultural Network Platform

Most devices or sensors are not built in such a way that they can connect to the internet for data sharing purposes. Local gateways, which serve as a bridge between all hardware devices and sensors for networking, protection and controllability, are designed to address this data sharing issue. Implementation of a greenhouse or field gateway increases the ability to automate and manage the greenhouse monitoring system in real time.

Hardware modules and cloud facilities are distributed, while fog computing incorporates capital. Fog computing lowers the cloud's computing burden and guarantees processing in real time. In this proposed network platform, the basic aim of fog computing is to optimise cloud computing resources' ondemand scalability by taking advantage of both cloud and edge computing. Several actuators, sensors, microcontrollers and central processing units have been introduced to track and feel different agricultural variables in hardware modules. Hardware modules are distributed and used for the development of services or processes on global or local networks.

Fast response time and the ability to share information are important for the implementation of smart farming. Two protocols, Representational State Transfer (REST) and Message Queuing Telemetry Transport, fulfil each of these criteria (fast response time and capacity to exchange information) (MQTT). It is more efficient for smart farming instead of using big data centre distributed system because it breaks up large computations into simple and smaller tasks such as: crop, temperature, nutrients, electricity, atmosphere, soil moisture, etc.

C. Topology and Protocols of the IoT Agricultural Network

The topology of the IoT agricultural network illustrates the arrangement of many elements of the IoT Agricultural Network and is an ideal smart farming scenario.

This ubiquitous agricultural solution turns numerous electronic devices such as smartphones, laptops and farm terminals into hybrid computing grids for storage space. Sensed information is then processed and stored, and for aggregation, stored data from various sensors and devices becomes useful. Farmers can track the various crop variables from anywhere in the entire field on the basis of aggregation and analysis. Moreover, for the streaming of agricultural images, topology consists of a proper network setup. 


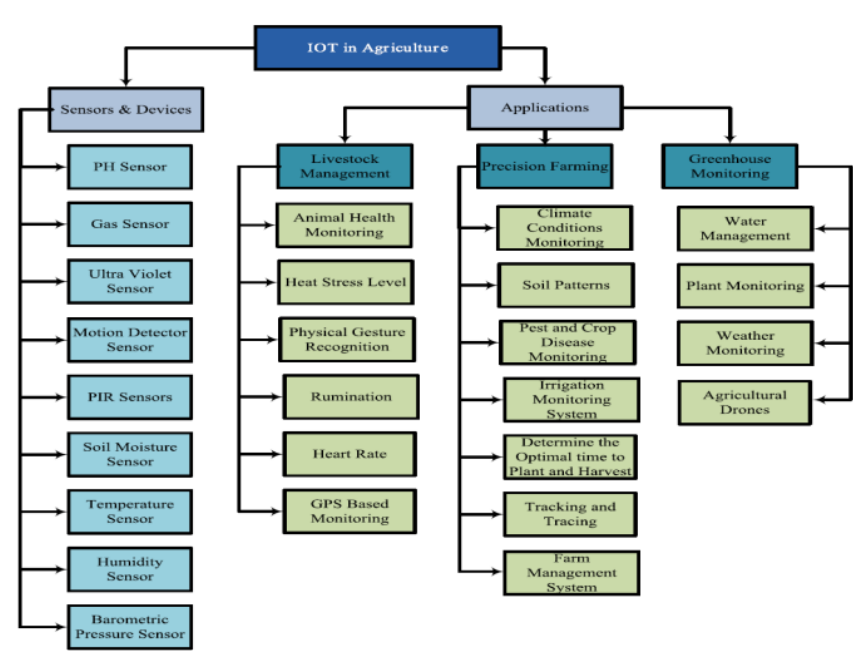

Fig. 3: Structure of IoT in agriculture

\section{CONCLUSION}

The IoT-based system of agricultural production has been founded on farmers' long-standing desire to ensure that their land remains viable in the future. It also addresses the needs and concerns of the community about nutritious food and the safety of the environment. An agricultural production system was developed using IoT technology for agricultural production and implemented as a GUI visualization programme. The IoT-based agricultural production system has strengthened farmers' ability to assess current conditions and forecast future harvests by analyzing the connection between crop statistical information and information on the agricultural climate. Furthermore, the quality of agricultural products can be improved because farmers track the entire cycle from seeding to sale using this agricultural production method based on the IoT.

In many ways, due to the Internet of Things, the internet is changing many facets of day-to-day agricultural operations. In agricultural operations, the advantages of IoT technology include, but are not limited to, waste reduction, improved pest control and increased productivity. IoT technology will be the key to lowering costs and optimizing yields from available resources as farmers continue to face rising costs and limited resources. The IoT is set to drive farming's future to the next level. Smart farming is already becoming more common among farmers, and thanks to agricultural drones and sensors, high tech farms are increasingly becoming the norm. IoTbased Smart Farming strengthens the entire agricultural system by real-time field monitoring. The Internet of Things in Agriculture, with the aid of sensors and interconnectivity, not only saved farmers' time but also minimized the extravagant use of resources such as water and electricity.

\section{REFERENCE}

[1] Abhishek, D., et al. 2019. Estimates for World Population and Global Food Availability for Global Health", Book chapter, The Role of Functional Food Security in Global Health, 2019, Pages 3-24

[2] Elder M., and Hayashi S. 2018. A Regional Perspective on Biofuels in Asia", in Biofuels and Sustainability, Science for Sustainable Societies, Springer, 2018

[3] Jayaraman, P.P., Yavari, A., Georgakopoulos, D., Morshed, A., and Zaslavsky, A. 2016. Internet of Things platform for smart farming: Experiences and lessons learnt. Sensors, 16(11):1884.

[4] Khattab, A., Abdelgawad, and K. Yelmarthi, K. 2016. Design and implementation of a cloud-based IoT scheme for precision agriculture," in Proc. 28th Int. Conf. Microelectron. (ICM), Dec. 2016, pp. 201-204.

[5] Madakam, S., Ramaswamy, R., and Tripathi, S. 2015. Internet of Things (IoT): A Literature Review. J. Comput. Commun. 3: 164-173.

[6] Verdouw, C., Sundmaeker, H., Tekinerdogan, B., Conzon, D., and Montanaro, T. 2019. Architecture framework of IoT-based food and farm systems: A multiple case study. Comput. Electron. Agric. 165: 104939.

[7] Zhao, J.C., Zhang, J.F., Feng, Y., Guo, J.X. 2010. The study and application of the IOT technology in agriculture. In Proceedings of the IEEE 2010 3rd International Conference on Computer Science and Information Technology, Chengdu, China, 9-11 July 2010; Volume 2, pp. 462-468.

[8] Farooq, M.S., Riaz, S., Abid, A., Abid, K., Naeem, M.A. A Survey on the Role of IoT in Agriculture for the Implementation of Smart Farming, IEEE Access, 2019.

[9] The Internet of Things. Submitted to asiapacteh. 